\title{
Rheology of Polytriazole/ZIF-8 Solutions and Dynamics of Mixed- Matrix Composite Films
}

\author{
Rebecca Esposito, Valentina Musteata, Stefan Chisca, and Suzana P. Nunes* \\ Cite This: https://doi.org/10.1021/acsapm.1c00501 \\ Read Online
}

ACCESS |

Lلll Metrics \& More

Article Recommendations

Supporting Information

ABSTRACT: Adhesion between fillers and a polymeric matrix is an essential characteristic for mixed-matrix membranes for molecular separation to avoid defects and cavities that would decrease selectivity. We demonstrate here how solution rheology can be an excellent tool to identity polymer-filler pairs with strong interaction. The investigated system was polytriazole with and without hydroxyl functionalization (PTA$\mathrm{OH}$ and PTA) and ZIF-8. ZIF-8/PTA-OH forms stable gels even with a small filler content, which was investigated under increasing shear rates and different temperatures, while analogous systems without $\mathrm{OH}$ functionalization do not gel. The investigations were supported by spectroscopic methods, electron microscopy, and dynamic mechanical analysis of the final solid film. While small ZIF contents lead to sensitive changes in the solution viscosity, only minor changes in the Fourier transform infrared and NMR

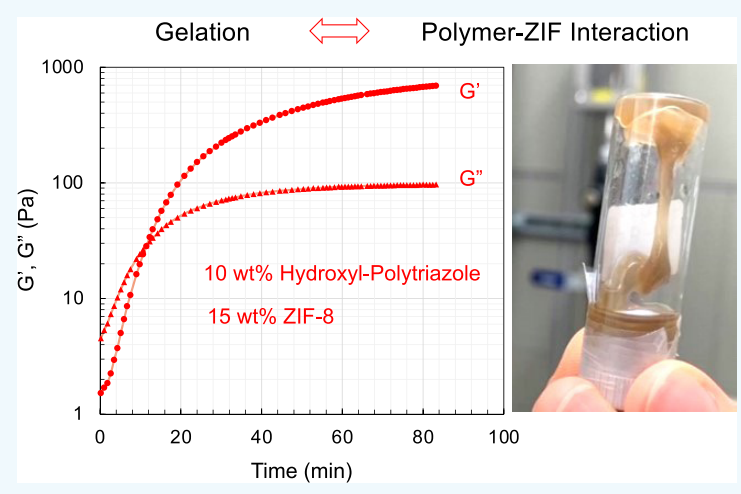
spectra were detected for the same system. The good adhesion between PTA-OH and ZIF- 8 could be seen by electron microscopy, while the analogous PTA/ZIF-8 composites provided films with poor matrix-filler adhesion and undesired cavities. The methods described in this work can be extended as useful tools in the development of composites such as mixed-matrix membranes applied to a large variety of currently available metal-organic frameworks and polymers.

KEYWORDS: rheology, polytriazole, ZIF-8, MOF, mixed-matrix, polymer-filler interaction

\section{INTRODUCTION}

Mixed-matrix membranes have raised expectations on the possibility of combining the intrinsic properties of polymers and inorganic or metal-organic fillers for decades. ${ }^{1-8}$ The main advantages are the excellent processability of polymeric materials, which can be manufactured into different configurations such as flat sheets and hollow fibers in continuous production, and the perspective of combining high selectivity and permeability for different separations. The interest in mixed-matrix membranes ${ }^{9-11}$ has grown even more with the advent of an infinite variety of finely tuned metal-organic frameworks (MOFs), which can be designed with different functionalities and pore sizes. ${ }^{12,13}$ However, the application of mixed-matrix membranes in industries did not reflect the expectations yet. Taking full advantage of exceptional fillers and polymers requires much more than choosing materials with the highest selectivity for a specific separation. A technically competitive membrane frequently consists of multilayers, with the most selective one being thinner than 1 $\mu \mathrm{m}$. Obtaining defect-free stable and scalable selective layers is essential. Tailoring the selective layer by choosing the right combination of materials has different challenges. Concentration, selectivity, and permeability are relevant parameters. The permeability and selectivity of a film constituted by blended materials are not the simple average values of each component. If the permeability of the matrix is too high, a permeant gas would preferentially pass through it without taking advantage of the filler characteristics, even if they would have excellent selectivity. To optimize the composition of an ideal binary system for multicomponent membranes aiming for gas separation, the Maxwell equation (see the Supporting Information) has been a classical tool, in analogy to the description of the conductivity in an electric circuit. ${ }^{14}$ However, the optimized combination of permeability and selectivity values predictable by the Maxwell equation is only valid if the dispersed and continuous phases are homogeneously distributed without defects or cavities between them. For mixed-matrix membranes, good adhesion between the filler and polymer matrix is essential, as illustrated in Figure 1. Poor adhesion leads to cavities around the filler particles, which would provide a preferential path for the permeants, erroneously indicating an apparent increase of permeability, however without reaching the expected selectivity. On the

Received: April 21, 2021

Accepted: September 21, 2021 


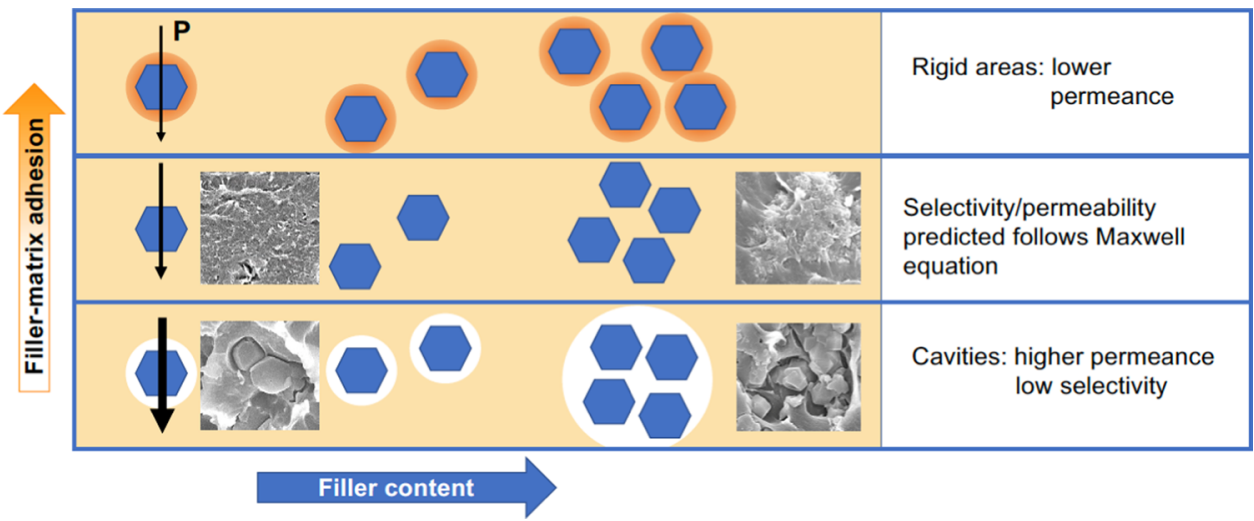

Figure 1. Schematic of filler content and filler-matrix adhesion effects on the separation properties of mixed-matrix membranes.

other hand, too strong interactions might lead to an increase in the rigidity of the polymer layer around the filler affecting the permeability. Verifying if a polymer/filler pair has sufficient adhesion for a good membrane is frequently confirmed after steps of optimization of membrane preparation and testing. A sufficient amount of polymer and filler is required. Having a simple method to effectively predict the interface characteristics $^{15}$ and screen different polymer-filler pairs even with small amounts of filler before investing time in the material preparation and membrane optimization would be very useful. Potential methods include the measurement of other properties indirectly affected by the polymer-filler interactions, for instance, spectroscopic methods, microscopy of fractured films, or mechanical characterizations. We compare some of the possibilities in this work. Membrane preparation is mostly a solution process. Strong interactions between fillers and specific functionalization groups of a polymer can lead to strong adhesion in the final composite film and have an impact on the rheology of the processing solution. In this work, we propose and explore the rheological investigation as a strong indicator of effective polymer-filler interaction to guide the preparation of defect-free polymeric films for membrane and other applications. Our focus here is the rheology of the solution, supported by chemical characterization. We demonstrate that the method can be successful even with low filler contents. We chose as model filler a zeolitic imidazole framework, ZIF-8, which has been frequently used for mixedmatrix preparation with promising performance, ${ }^{16-24}$ having zinc ions as the coordination metal and imidazole linkers. A list of previously prepared membranes using ZIF in a combination of different polymers is shown in Supporting Information Table S1. ZIF is a commercial product and easily available. An even more important reason for using ZIF is its imidazole functionalization that can be a source of interaction with the chosen polymer in this work, which is a fluorinated polytriazole with hydroxyl groups (PTA-OH). PTA-OH has been investigated by our group for different membrane applications and has demonstrated high stability, easy processability, and versatility in terms of functionalization with potential interaction sites provided by hydroxyl and amino groups. ${ }^{25-27}$ The functionalizations of PTA-OH could in principle also be a source of interaction with the zinc atoms of ZIF. ZIF-8 would therefore act as a noncovalent cross-linker, enabling the formation of stable polymer gels in different organic solvents with a characteristic rheological behavior.

Gelation of polymer solutions occurs in different forms, and understanding the mechanism has been a topic of theoretical and experimental work by different groups with classical models proposed early by Flory, ${ }^{28}$ Stockmayer, $^{29}$ and De Gennes. ${ }^{30}$ Gelation can be observed during polymerization, as the chain grows to a large molecular weight or even cross-links as a result of covalent bonding, while still surrounded by solvent. Physical gelation can result from partial crystallization, coil-helix transitions, or by the thermodynamic deterioration $^{31}$ of the solvent quality by changing the temperature, leading to thermal reversible gels. Thermodynamic interactions can be governed by hydrophobic-hydrophilic contacts, polarity, and hydrogen bonding. Ion complexation can also promote gelation. Polymer gels have been also considered as percolated colloidal particles. $^{32}$

The method proposed here could be extended to new functionalized polymers and MOFs frequently synthesized in small amounts to evaluate their potential before investing in the scaling up of the material synthesis and membrane fabrication.

\section{EXPERIMENTAL SECTION}

2.1. Materials. $N, N$-Dimethylformamide (DMF), $N, N$-dimethylacetamide (DMAc), 2-methylimidazole zinc salt, and ZIF-8 (Basolite Z1200) were purchased from Sigma-Aldrich. Fluorinated polytriazole (PTA) and the corresponding hydroxyl-functionalized polytriazole (PTA-OH) were synthesized in our lab, as reported before and described in the Supporting Information. ${ }^{25-27,33}$

2.2. Rheological Measurements. The rheological properties of freshly prepared solutions of PTA-OH with different ZIF-8 contents were investigated on an AR-2000ex rheometer (TA Instruments Ltd, New Castle, DE), as detailed in the Supporting Information. If not otherwise specified, the final polymer solution contained 10 wt \% PTA-OH.

2.3. Chemical and Morphological Characterization. The Fourier transform infrared (FTIR) spectroscopy investigation was done with a Nicolet 6700 FT-IR System. Nuclear magnetic resonance (NMR) spectroscopy investigations were conducted in solution and in solid samples. The spectra were obtained on a Bruker Advance III 400. The mass ratio between the polytriazole and the zinc acetate dihydrate or 2-methylimidazole was 6:1 (mg). One-dimensional ${ }^{13} \mathrm{C}$ CP/MAS solid-state NMR spectra were recorded on Bruker AVANCE III spectrometers operating at 400 and/or $600 \mathrm{MHz}$ resonance frequencies for ${ }^{1} \mathrm{H}$.

2.4. Scanning Electron Microscopy (SEM). For the morphological analysis, dry film samples with different ZIF-8 contents, prepared as detailed in the Supporting Information, were fractured in liquid nitrogen, sputter-coated with iridium using a Quorum Q150TES equipment, and imaged on a Nova Nano FEI field emission scanning electron microscope. The voltage was in the range of $3-5 \mathrm{kV}$, and the working distance was in the range of $3-5 \mathrm{~mm}$. 
PTA

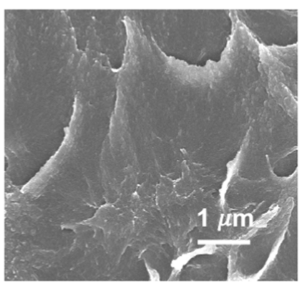

PTA-OH

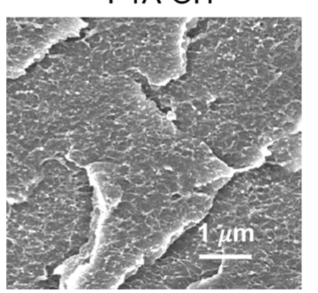

10 wt $\%$ ZIF-8

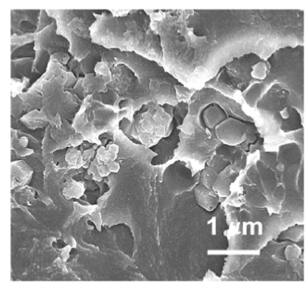

10 wt \% ZIF-8

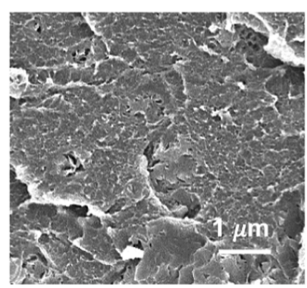

$20 w t \%$ ZIF-8

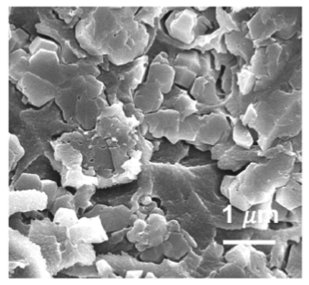

20 wt \% ZIF-8

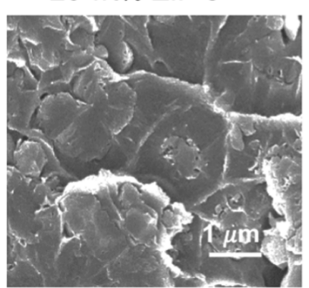

30 wt $\%$ ZIF-8

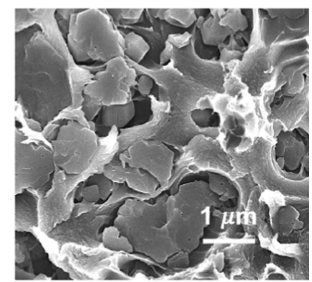

30 wt\% ZIF-8

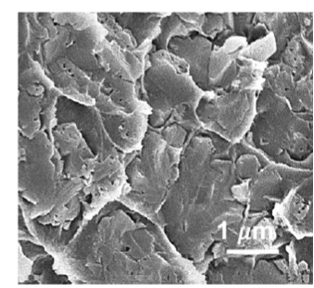

Figure 2. Cross-sectional SEM images of PTA (top) and PTA-OH (bottom) films with different contents of ZIF-8.
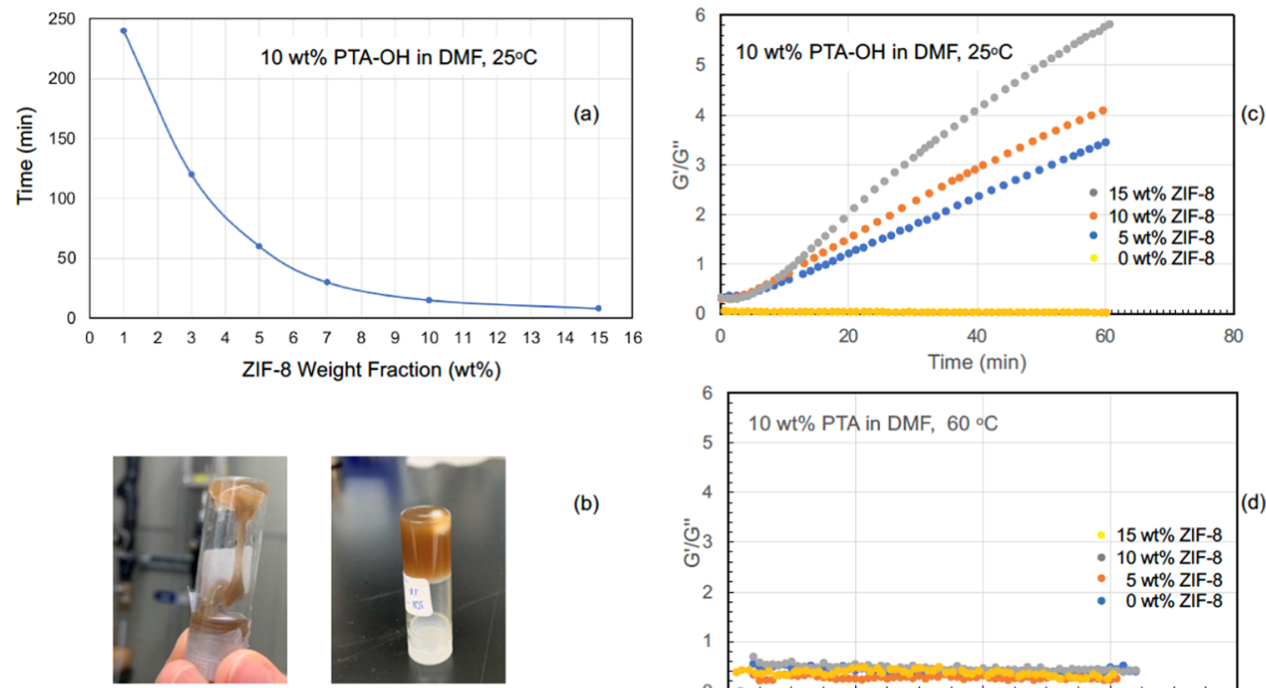

(b)

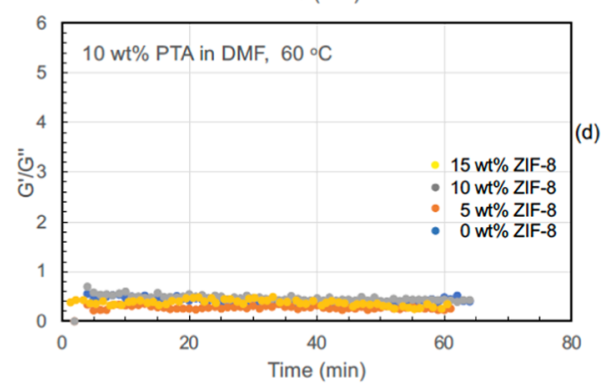

Figure 3. Gelation of PTA-OH and PTA solutions in DMF: (a) Time till gelation for 10 wt \% PTA-OH solutions as a function of ZIF-8 content. (b) Photographs of gelling ZIF-8/PTA-OH solutions in DMF (complete gelation on the right). (c) Ratio between storage $\left(G^{\prime}\right)$ and loss $\left(G^{\prime \prime}\right)$ moduli as a function of time of solutions with $10 \mathrm{wt} \%$ PTA-OH and different ZIF-8 contents. (d) Ratio between storage $\left(G^{\prime}\right)$ and loss $\left(G^{\prime \prime}\right)$ moduli as a function of time of solutions with $10 \mathrm{wt} \%$ PTA and different ZIF- 8 contents.

2.5. X-ray Diffraction (XRD). XRD measurements were performed on a Bruker D8 Advance X-ray diffractometer using Cu $\mathrm{K} \alpha \alpha_{1}$ radiation $(\lambda=1.5406 \AA)$. Samples were measured in the $2 \theta$ angle scan range of $5-60^{\circ}$ using a step size of $0.2^{\circ}$ and a scanning rate of $1^{\circ} / \mathrm{min}$.

2.6. Mechanical Characterization. Dynamic mechanical analysis (DMA) was carried out with a Discovery DMA 850 (TA Instruments). Details are shown in the Supporting Information.

\section{RESULTS AND DISCUSSION}

Fluorinated polytriazole and ZIF-8 were expected to form highly homogeneous composite films due to the similarity between the ZIF-8 linker, imidazole, and triazole group in the polymer backbone. In the case of PTA (without hydroxyl groups), although the films are visually homogeneous, when their cross section was imaged by SEM, ZIF- 8 particles could be seen clearly detached from the matrix, as shown in Figures 2 and S1, an indication of relatively poor adhesion. Cavities around the filler particle are known to contribute to weaken the mechanical properties and promote the formation of nonselective paths for the transport of molecules. These are detrimental characteristics for systems aiming applications as membranes. The cavities are even more evident at a higher ZIF-8 content. Fortunately, the cavities are practically absent in composites prepared with the analogous polymer PTA-OH, with almost the same chemical structure but functionalized with $\mathrm{OH}$ groups.

We observed that solutions prepared with PTA-OH gel after adding small amounts of ZIF-8, while analogous solutions with PTA do not gel. Figure $3 \mathrm{a}$ shows that the time for gelation of 10 wt \% PTA-OH solutions in DMF is highly dependent on their content of ZIF-8. In this plot, the system was considered fully gelled when no flow could be visibly detected in upsidedown vials (see Figure $3 \mathrm{~b}$ ). More precisely, we measured the storage $\left(G^{\prime}\right)$ and loss $\left(G^{\prime \prime}\right)$ moduli as a function of time for solutions with 10 wt \% PTA-OH and different ZIF- 8 contents 


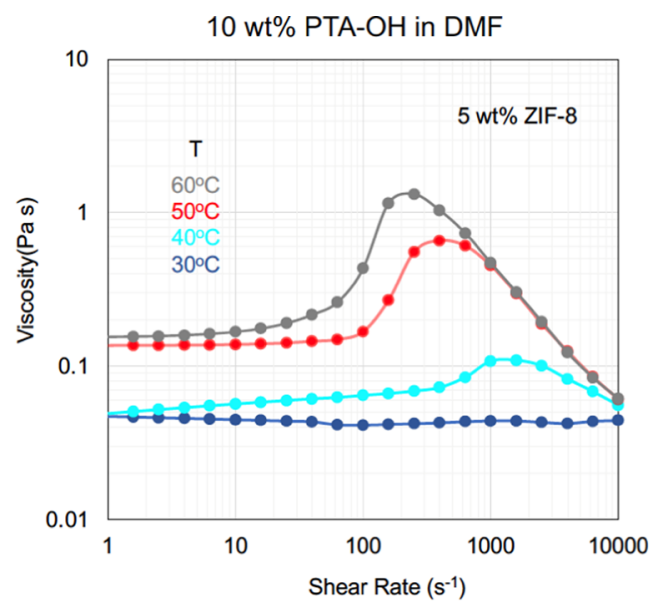

(a)

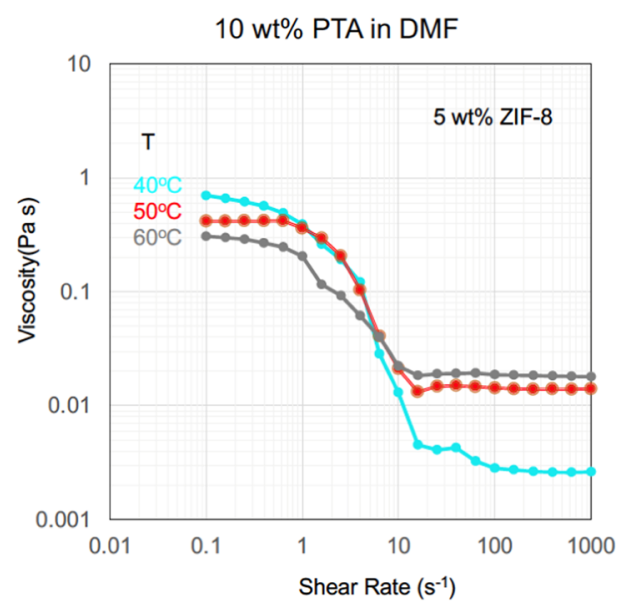

(b)

Figure 4. (a) Viscosity of freshly prepared $10 \mathrm{wt} \%$ PTA-OH solutions in DMF with $5 \mathrm{wt} \% \mathrm{ZIF}-8$ (relative to the polymer content) as a function of shear rate and temperature. (b) Viscosity of freshly prepared 10 wt \% PTA solutions in DMF with 5 wt \% ZIF-8 (relative to the polymer content) as a function of shear rate and temperature.

(Figure 3c). Initially, for all solutions $G^{\prime \prime}>G^{\prime}$, similar to solutions without ZIF-8 (Figure S2), the $G^{\prime} / G^{\prime \prime}$ ratio is $<1$. When no ZIF-8 is added, practically no significant change of viscosity or $G^{\prime} / G^{\prime \prime}$ with time was observed. This indicates the viscous fluidic behavior of the solutions. For samples with 515 wt \% ZIF-8, above $12 \mathrm{~min}$ (Figure 3c), $\mathrm{G}^{\prime}$ becomes larger than $G^{\prime \prime}$, demonstrating that a gel-like behavior is developed. The time corresponding to the crossing of the $G^{\prime}$ and $G^{\prime \prime}$ curves $\left(G^{\prime} / G^{\prime \prime}=1\right)$ increases as $5>10>15$ wt $\%$. We performed the same experiment, substituting PTA-OH by the analogous nonfunctionalized polytriazole. In this case, due to the lack of solubility at room temperature, the experiments were conducted at $60{ }^{\circ} \mathrm{C}$. As seen in Figure $3 \mathrm{~d}$, no gelation was observed. $G^{\prime} / G^{\prime \prime}$ remained constant with time and smaller than 1 , characterizing a viscous behavior.

The rheology of solutions with 10 wt \% PTA-OH (or PTA) and different contents of ZIF- 8 were then investigated in detail. Figure S3a shows first how the viscosity of PTA-OH solutions without ZIF-8 depends on shear rate. As expected, at a low shear rate, the viscosity is constant and the solutions have a Newtonian behavior. As the shear rate increases, a shear thinning is observed, which is usually understood as the elongation or partial disentanglement of polymer coils in solution. ${ }^{34}$ The inverse of the shear rate corresponding to the initial viscosity decrease can be correlated to the relaxation time of the system. For weight fractions 10, 12, and 15 wt \%, the values at which the shear thinning initiates at $25{ }^{\circ} \mathrm{C}$ were 40,400 , and $600 \mathrm{~s}^{-1}$, with the corresponding relaxation times of $0.025,0.0025$, and $0.0017 \mathrm{~s}$. The addition of only $3 \mathrm{wt} \%$ ZIF-8 (relative to the polymer content) to the PTA-OH solution causes gelation with a 4 orders of magnitude increase in viscosity, as seen in Figure S3b. By fixing the polymer fraction in $10 \mathrm{wt} \%$, it was possible to observe in Figure S3c an even higher increase of viscosity as the ZIF- 8 content increased from 3 to 15 wt \%.

An initial time is required for ZIF- 8 and polymer chains to associate. ZIF- 8 acts as a physical cross-linker as the contact between more sites will be formed. The nature of this interaction will be later discussed based on spectroscopic measurements.
By investigating the viscosity of freshly prepared $10 \mathrm{wt} \%$ PTA-OH solutions in DMF with 5 wt \% ZIF-8 (relative to the polymer content) as a function of temperature, we see in Figure $4 \mathrm{a}$ a clear increase in viscosity (shear thickening) before shear thinning is initiated. The shear rate at which the maximum occurs depends on temperature. For $30{ }^{\circ} \mathrm{C}$ or below, the maximum is probably out of the measurement range of the rheometer, with a slight increase of viscosity starting just below $10000 \mathrm{~s}^{-1}$. As the temperature increases to 40, 50, and $60{ }^{\circ} \mathrm{C}$, a maximum is clearly seen, occurring, respectively, at 1100,400 , and $240 \mathrm{~s}^{-1}$. Shear thinning in polymers solutions without additives is understood as simply related to the disentanglement of polymer coils under shearing. ${ }^{34}$ Shear thickening and thinning reflect the friction between chain segments and are therefore influenced by the quality of the solvent, which may change with temperature. ${ }^{35}$ In the system represented in Figure 4, the shear thickening and thinning start at a lower shear rate at a higher temperature. A higher mobility of the polymer coils at a higher temperature would explain this behavior with less shearing being enough to disentangle coils under flow. Shear thickening is an indication of the presence of a transient network, caused by the stretching of potential intersegment bridges or by the formation of additional bridges. $^{36,37}$ In our system, bridges are built by the strong interaction between specific groups in the polymer chains and ZIF-8. The kind of interaction is discussed later, based on spectroscopic data. In Figure 4, the shear thickening is more pronounced at higher temperatures, with differentiated shear thickening for systems above $40{ }^{\circ} \mathrm{C}$. However, one should keep in mind that the viscosity values at a low shear rate are in the range of $0.05-0.15 \mathrm{~Pa} \mathrm{~s}$. Under these conditions, the interaction with ZIF-8 is still being built. The results in Figure 4 were obtained with freshly prepared solutions, immediately after mixing the PTA-OH solution and the ZIF-8 dispersion, when gelation still did not start. Figure S4 presents Arrheniuslike plots of viscosity as a function of the inverse of temperature, at different shear rates. The slopes are steeper at 252 and $398 \mathrm{~s}^{-1}$, range at which shear thickening is more pronounced, indicating a relatively high activation energy to break the transient network built by the entangled polymer chains with incipient ZIF-8 connections. 


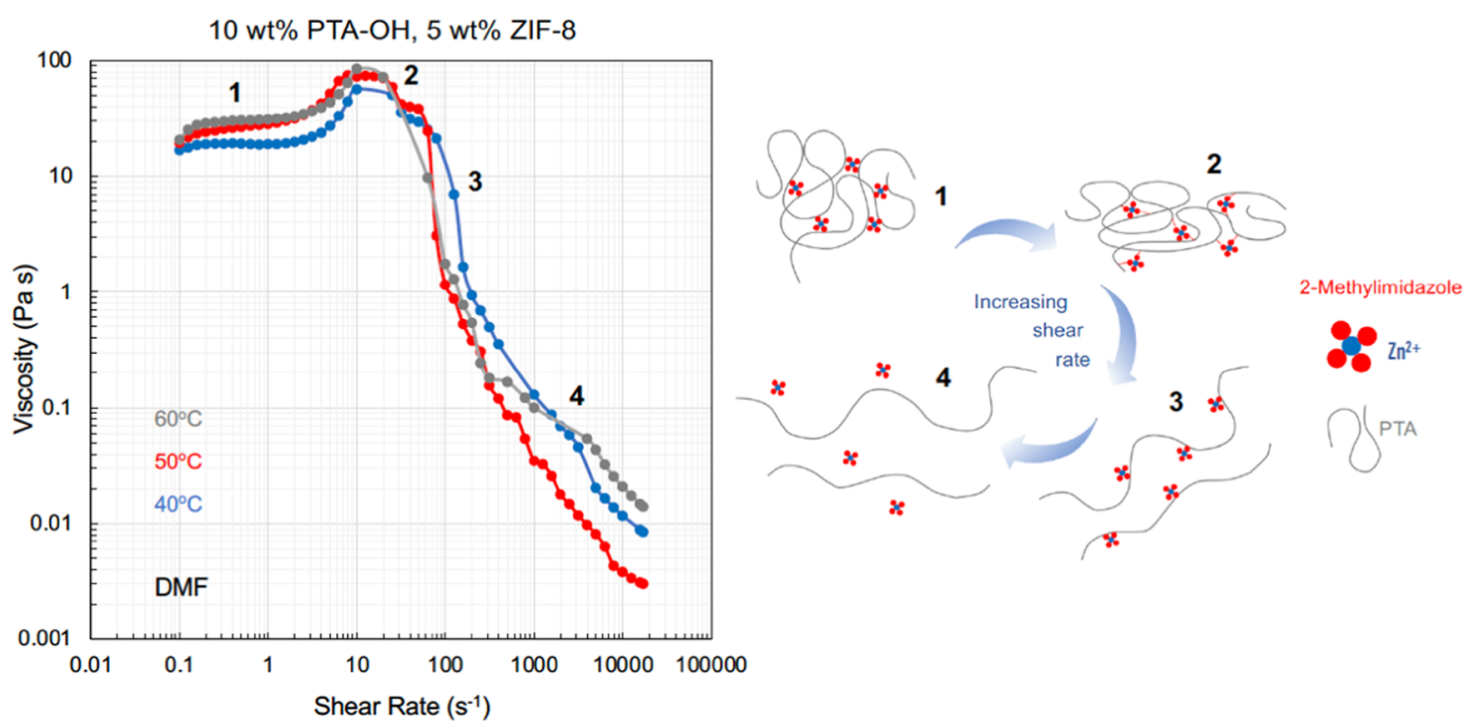

(a)

(b)

$10 w t \%$ PTA-OH, $25^{\circ} \mathrm{C}$

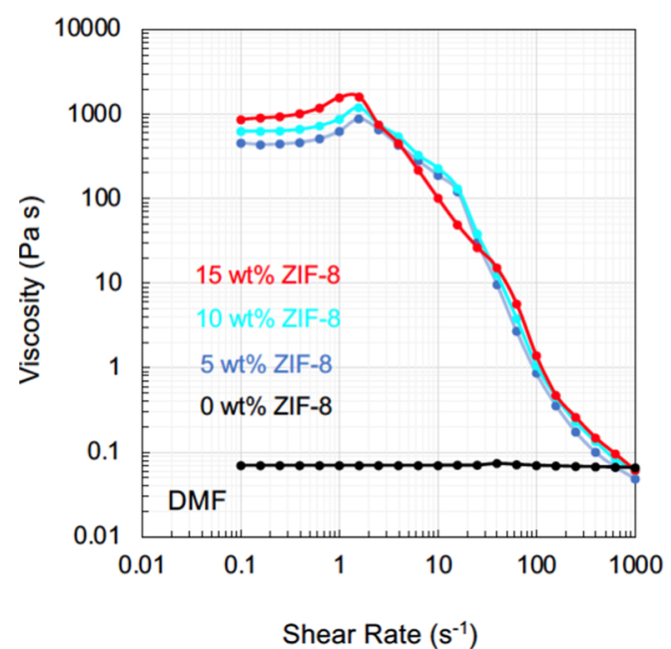

(c)

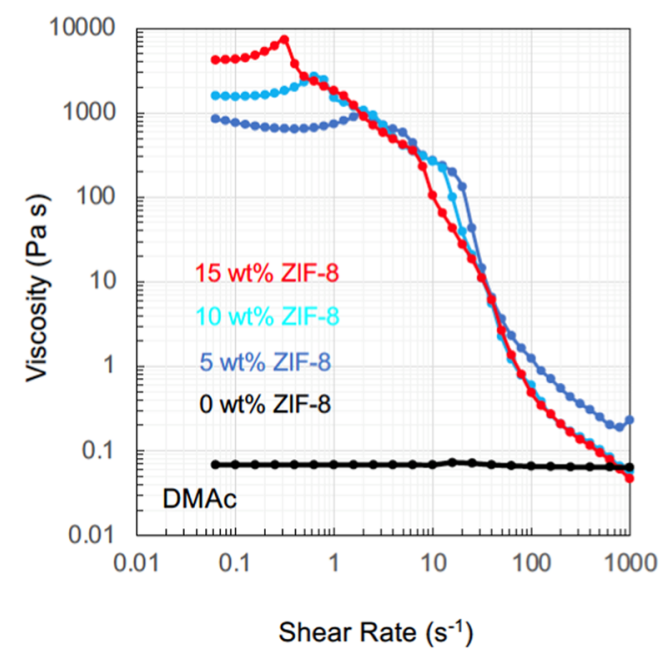

(d)

Figure 5. (a) Viscosity as a function of shear rate at different temperatures of 10 wt \% PTA-OH solutions in DMF containing 5 wt $\%$ ZIF-8 (relative to the polymer content), after the samples were partially gelled. (b) Simplified scheme of polymer-chain disentanglement and ZIF-8 bridge disconnection as the shear rate increases. (c, d) Viscosity as a function of shear rate of partially gelled 10 wt \% PTA-OH solutions in (c) DMF and (d) DMAc containing up to $15 \mathrm{wt} \%$ ZIF-8 (relative to the polymer content).

Freshly prepared 10 wt \% PTA (without OH functionalization) solutions in DMF with 5 wt \% ZIF-8 do not suffer shear thickening in the investigated temperature range of $40-60{ }^{\circ} \mathrm{C}$ (Figure $4 \mathrm{~b}$ ). In contrast, a drastic shear thinning is observed above $1 \mathrm{~s}^{-1}$. At $40{ }^{\circ} \mathrm{C}$, this is even more pronounced. In this case, a small viscosity increase was observed above $30 \mathrm{~s}^{-1}$, which could indicate phase separation.

The rheology of fluids with dispersed fillers without preferential interactions has been classically understood with the help of simple models ${ }^{37}$ such as the Einstein equation (Supporting Information). But this is a simplified approach. In our system, the ZIF-8 particles are much more than inert fillers added to the polymer solution. They are highly interactive and act as knots tightening macromolecular segments together and promoting gelation. Understanding the rheology of the system requires considering additional aspects that give important insight into the interactions between filler and polymers and might enable us to predict how the filler-matrix adhesion in a film will be.

Figure 5 reports the rheological investigation of the systems after the gelation of ZIF-8/PTA-OH was initiated. While no gelation is observed for samples without ZIF-8, with the viscosity remaining lower than $0.1 \mathrm{~Pa} \mathrm{~s}$ (Figure S3a), the viscosity values are orders of magnitude larger, above $400 \mathrm{~Pa} \mathrm{~s}$, 

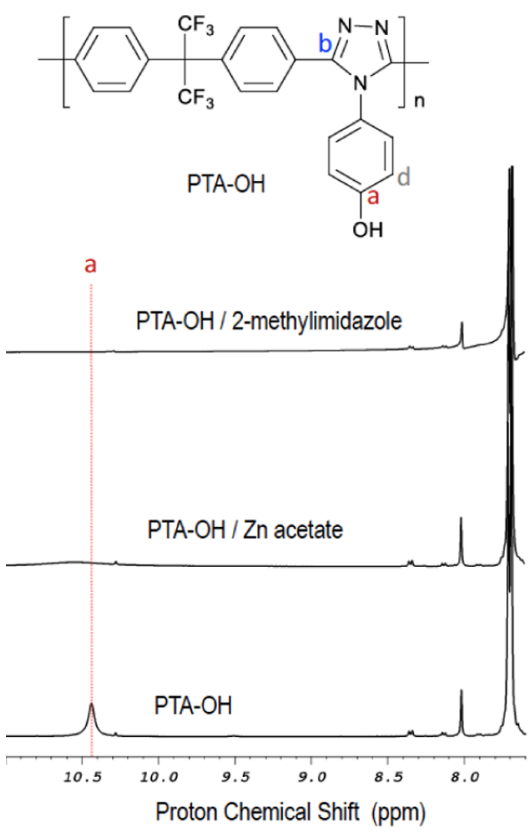

(a)

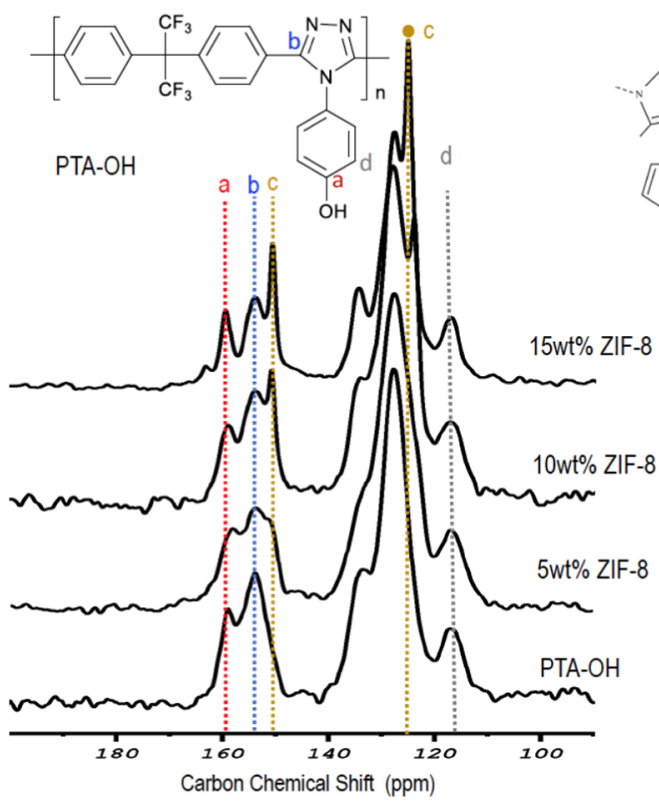

(c)

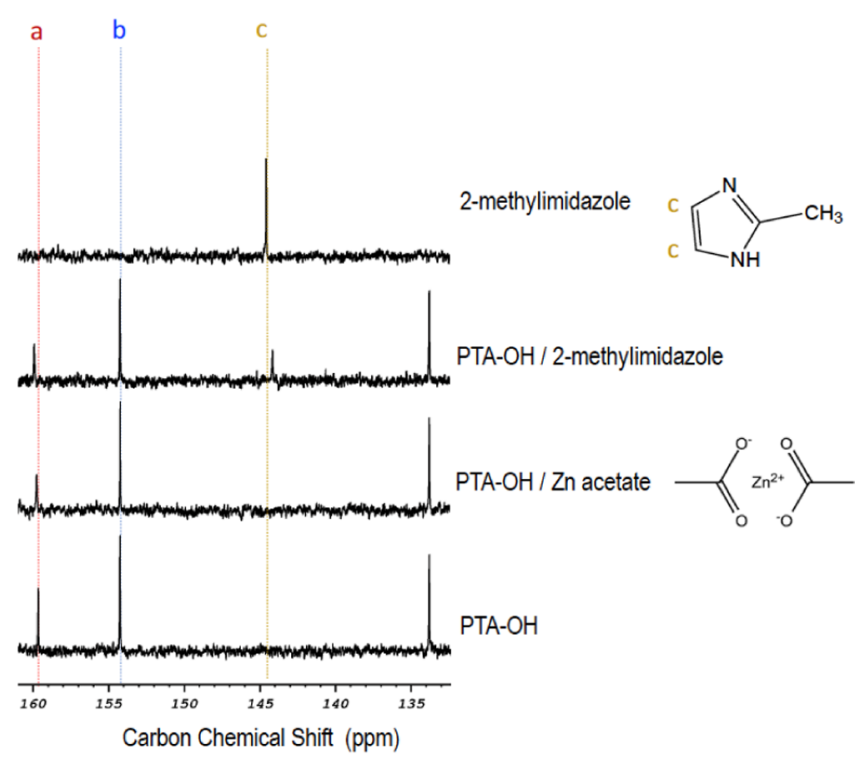

(b)
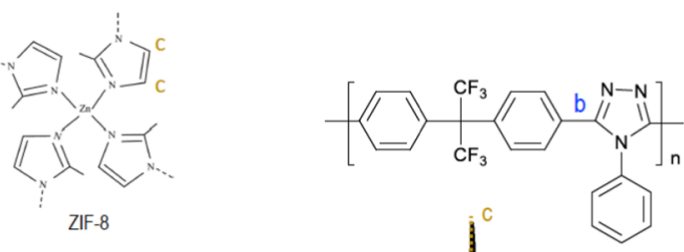

PTA

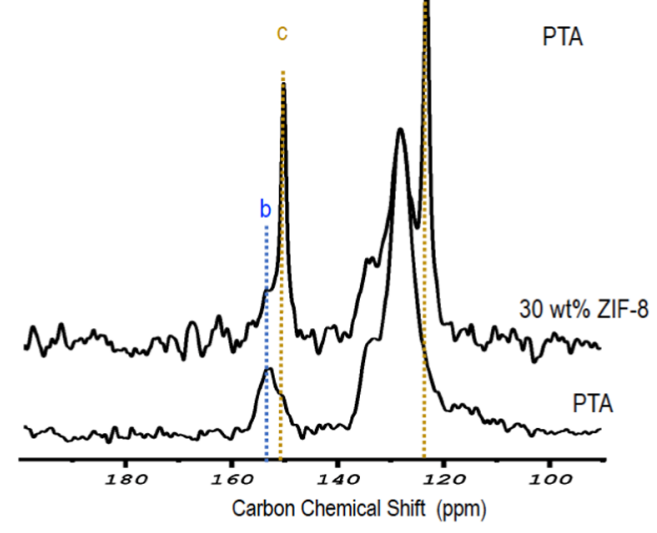

(d)

Figure 6. (a, b) Spectroscopic characterization of PTA-OH solutions in deuterated DMF: (a) ${ }^{1} \mathrm{H}$ NMR and (b) ${ }^{13} \mathrm{C}$ NMR spectra. The weight ratio of zinc acetate or 2-methylimidazole to PTA-OH added to the solution was 1:6. (c, d) Solid ${ }^{13} \mathrm{C}$ NMR spectra of films prepared from (c) PTA-OH and (d) PTA solutions with different contents of ZIF-8.

for systems with $>5 \mathrm{wt} \%$ ZIF-8. For these systems, the shear thickening is higher than $400 \mathrm{~Pa} \mathrm{~s}$ (Figure 5). The plots in Figure 5 have multi-inflections first with a light shear thickening, followed by a sharp shear thinning. This behavior indicates the presence of transient networks with a polydisperse set of bridges with different strengths and relaxation times. The first shear thickening probably reflects the tendency of the macromolecule to disentangle, driven by the increasing shear rate (as depicted in Figure 5b), at the same time suffering a strong resistance to break the strong bridges between the polymer and ZIF-8. As the shear rate is increased, these bridges holding the entangled coils together break, the friction between segments decreases, as well as the viscosity decreases. As the shear rate continues to increase, the remaining links between ZIF-8 particles and the already disentangled macromolecules are also disrupted. As seen later, 
bridges might be formed by strong hydrogen bonds between the polymer and the linker or potentially by competing to coordinate with the zinc ions.

The first shear thickening events in Figure 5a,c for solutions in DMF are around $1.5 \mathrm{~s}^{-1}$, independently of the ZIF-8 content in the range between 5 and $15 \mathrm{wt} \%$. For solutions in DMAc in Figure $5 \mathrm{~d}$, the corresponding maxima are significantly shifted from 0.3 to 0.6 and $2 \mathrm{~s}^{-1}$, when decreasing the ZIF- 8 content from 15 to 10 and 5 wt \%. At least one additional inflection point can be seen at a higher shear rate in plots for solutions in DMF $\left(20-40 \mathrm{~s}^{-1}\right)$. In DMAc, additional steps around 6-15 and $15-30 \mathrm{~s}^{-1}$ were also observed. DMF and DMAc have similar structures, differing by a hydrogen or methyl group linked to the carbonyl group, respectively. Particularly, DMF can form strong directional $\mathrm{C}=\mathrm{O}-\mathrm{H}-\mathrm{N}$ bonds. ${ }^{38}$ DMF might compete more for $\mathrm{H}$-bonds or other forms of association with the polymer and/or ZIF-8 imidazole linkers than DMAc. It is possible that DMF would compete to interact with PTA-OH or with ZIF-8, while in DMAc, this would happen much less. One kind of interaction might be by hydrogen bonding. This affects the strength and density of "bridges" between polymers segments and ZIF-8. The polymer-ZIF-8 interactions responsible for the formation of a gel network with characteristic high viscosity values are therefore more dependent on the ZIF-8 concentration in DMAc solutions than in DMF.

Figure 5a shows the rheological behavior of partially gelled 10 wt \% PTA-OH solutions in DMF with 5 wt \% ZIF-8 at different temperatures. The data were collected at an earlier stage of gelation than for Figure $5 \mathrm{c}$. The maximum relative to shear thickening occurs around $2 \mathrm{~s}^{-2}$ at all temperatures. A second event more affected by temperature is seen at 50-80 $\mathrm{s}^{-1}$, occurring at a higher shear rate at a lower temperature, at which it is also more evident. A final step is seen above $500 \mathrm{~s}^{-1}$, more pronounced at a higher temperature. PTA without $\mathrm{OH}$ functionalization is much less soluble in DMF. It could be dissolved at $60{ }^{\circ} \mathrm{C}$. A few experiments could be performed only at $60{ }^{\circ} \mathrm{C}$ with 10 wt \% PTA in DMF with 5 wt \% ZIF-8 relative to the polymer content. Measurements performed at $10 \mathrm{~s}^{-1}$ or above indicated viscosity around $0.02 \mathrm{~Pa}$ s, which is 4500 -fold lower than values measured for PTA-OH in DMF with 5 wt \% ZIF-8 at this shear rate, corresponding to the maximum value in Figure 5a, confirming that gelation did not take place in the absence of $\mathrm{OH}$ functionalization.

The strong gel character of ZIF-8/PTA-OH solutions with intense storage modulus in DMF is typically demonstrated in Figure S2 for solutions in DMF. For the polymer films without ZIF-8, $G^{\prime}$ at $10 \%$ oscillation strain was below $1 \mathrm{~Pa}$. Above $100 \%$ oscillation strain, the gel network of composite films is disrupted, and the system acquires a more fluidic behavior with higher loss moduli.

As the strong interaction and consequent gelation were confirmed by rheological experiments, we performed a spectroscopic investigation to identify the groups responsible for the interaction. While ZIF-8 is constituted by $\mathrm{Zn}^{2+}$ coordinated by four 2-methylimidazole linkers, the bridge with PTA-OH could, in principle, occur by interaction through the potential presence of some noncoordinated zinc atoms or through interactions with the linker. PTA-OH has triazole rings and $\mathrm{OH}$ groups, which would be the most probable interactive sites. FTIR and NMR peaks assignments followed the literature for analogous functionalization. ${ }^{39,40}$ FTIR spectra are reported in the Supplementary Information (Figure S5). A clear increase of peaks relative to the imidazole linker and coordinated metal was observed due to the addition of ZIF-8 to the system, but this did not lead to a visible shift or quantitative confirmation of interaction between specific groups in the polymer and filler, indicating that the sensibility of FTIR spectroscopy for this purpose might not be enough. NMR was then applied. Figure 6 (and Figures S6-S9) shows ${ }^{1} \mathrm{H}$ NMR and ${ }^{13} \mathrm{C}$ NMR spectra of PTA-OH solutions in deuterated DMF compared to solutions to which zinc acetate or 2-methylimidazole were added (polymer:additive weight ratio, 6:1). It can be seen in Figure $6 a$ that the peak at 10.45 $\mathrm{ppm}$, relative to the free $\mathrm{OH}$ functionalization, disappeared in the presence of additives, suggesting that it is now committed to a strong interaction, which can, in principle, happen both with the metal and with the linker. The potential coordination with the zinc ions is due to the acidic nature of the phenolic proton and the strong electron-withdrawing character of the triazole rings. ${ }^{41}$ From the ${ }^{13} \mathrm{C}$ NMR spectra in Figure $6 \mathrm{~b}$, a minor shift of the peak relative to the carbon bonded to the $\mathrm{OH}$ group could be observed when 2-methylimidazole was added. From the solid ${ }^{13} \mathrm{C}$ NMR spectra in Figure $6 \mathrm{c}$, minor shifts in the peak relative to the carbon bonded to the $\mathrm{OH}$ group were seen with the addition of 5 and $10 \mathrm{wt} \%$ ZIF-8, and a shift relative to carbons from the imidazole ring was seen with 15 wt \% ZIF-8. No significant shift could be seen in the PTA spectra in Figure 6d, evidencing that the $\mathrm{OH}$ functionalization is the main site in the polymer responsible for a strong adhesion between filler and matrix and for the gelation of the hybrid solution. Triazole could, in principle, coordinate with free $\mathrm{Zn}^{2+}$, by acting as a Lewis base in the same form as imidazole. The basicity of triazole is however lower ${ }^{42}$ and should not be enough to compete with the already formed imidazole- $\mathrm{Zn}^{2+}$ complex of the added ZIF- 8 . On the other hand, the phenol groups of PTA-OH can form strong hydrogen bonds to Lewis bases, especially those with an electron-rich donor atom like imidazole. This has been investigated early, for instance, by Epley and Drago ${ }^{43}$ using calorimetry. Therefore, we believe that the phenol-imidazole interaction is the main reason for the gelation in this work.

Figure S10 shows X-ray diffractograms of PTA-OH, a series of films with increasing ZIF-8 contents and pure ZIF-8 as well. The XRD patterns show that all of the peaks of pure ZIF- 8 are present in the ZIF-8/PTA-OH films, indicating that the crystal structure of ZIF-8 was retained. However, a clear shift of the ZIF-8 peak at $7.5^{\circ}$ is seen when dispersed in PTA-OH for all films corresponding to a distance increase from $16.29 \AA$ to 17.70 A. Possibly, the matrix-filler interaction can cause this change, as it is already known that under specific circumstances like very high pressure and gas adsorption uptake, ZIF-8 can expand due to the flexibility of the organic linker. ${ }^{44}$

A good interaction between filler and polymer should contribute to satisfactory mechanical properties. Figure S11a shows stress-strain curves of films prepared with increasing ZIF- 8 contents, and additional characterization is reported in Table S2. The integration of stress-strain curves led to the toughness values in Figure $S 11 \mathrm{~b}$. The toughness and tensile strength decrease with the incorporation of ZIF-8. One potential reason might be the local aggregation of the filler that could to be minimized. But independent of that, the toughness and processability of polymers are known to be better than those of MOFs. Polymers are even used to enable MOF processability in the form of fibers. ${ }^{45}$ Although lower than that for the pure polymer, the values for PTA-OH/ZIF-8 


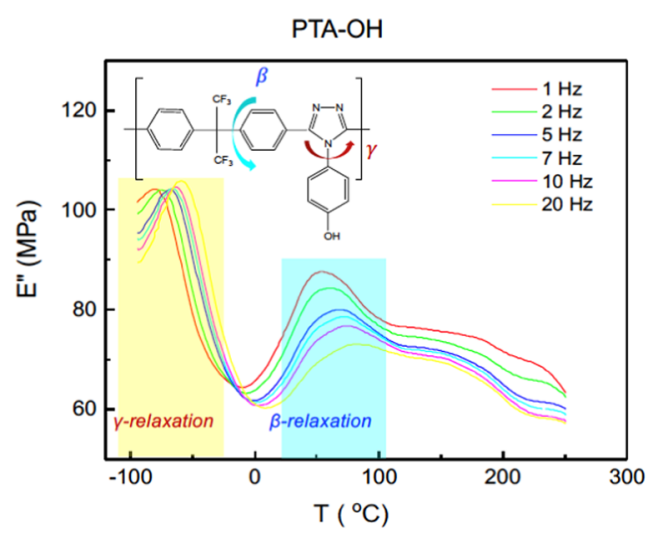

(a)

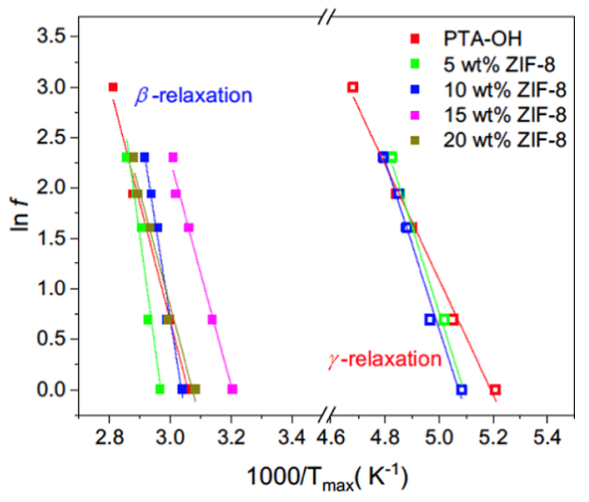

(b)

Figure 7. Dynamic mechanical analysis: (a) relaxations of PTA-OH visualized in curves of loss moduli as a function of temperature; (b) logarithm of the frequency corresponding to the maximum of the $E^{\prime \prime}(T)$ as a function of the inverse of temperature for films with different ZIF-8 contents. The activation energy values for the relaxations are estimated by the slopes of each curve.

composites are still high compared to analogous films prepared with other polymers, including other examples with $\mathrm{OH}$ functionalization. $^{46,47}$ Creep tests in Figure S11c show that samples submitted up to $0.7 \%$ strain could be recovered without permanent deformation. For the preparation of composite membranes for gas separation, the selective layer is ultrathin and deposited on a thicker, porous support. Therefore, the mechanical stability of the selective material becomes less important. The adhesion needs to be enough to avoid the formation of cavities between the polymer and the filler but does not need to secure high toughness.

More than the mechanical characterization reported in Figure S11 and Table S2, the DMA is sensitive to variations of the viscoelastic properties of the final solid films. In analogy to that analyzed in solution, by submitting the system to oscillating stresses, information on the molecular motions during the specimen heating could be obtained. ${ }^{48,49}$ The effect of ZIF-8 loading on the molecular dynamics of PTA-OH films was in this form investigated. As shown in Figures 7 and S12, the increase of the sample mobility during heating is accompanied by mechanical relaxations, which is manifested as a drop of the storage modulus as the polymer stiffness decreases. A maximum loss modulus is observed because a higher amount of energy is lost due to the increased internal motion within the material. To distinguish between mechanical relaxations correlated with polymer mobility and other processes that might occur, such as cross-linking, thermal rearrangement, ${ }^{49}$ or removal of trapped solvent vapors, ${ }^{50}$ we performed the DMA experiments at several frequencies. The reasoning is that mechanical relaxations obey the well-known time-temperature superposition principle: when a higherfrequency stress is applied, the relaxation shifts to a higher temperature, which basically means that at higher temperatures, the relaxation time is smaller. Based on this rule, in the investigated range, we identified two relaxations for ZIF-8/ PTA-OH films, which are seen in Figures 7 and S12 and discussed in the next paragraphs, while for the process around $200{ }^{\circ} \mathrm{C}$, we could exclude a relaxation underlying mechanism. This transition could involve the release of any residual solvent trapped within the ZIF- 8 particles, as reported before for ZIF$8 /$ Matrimid composites. ${ }^{50}$ Starting from lower temperatures, as the material heats and expands, there is an increase of free volume, which allows bending and stretching of bonds in the polymer-chain and side-chain movements. ${ }^{51}$ This is a $\gamma$ relaxation, and for polyimides, it encompasses limited length scale having a local character. It has been previously shown for other systems that it may involve associations with water. $^{48,49,52}$ The free volume continues to increase with a further increase of temperature. The localized groups in the backbone gain mobility and also encompass side-group movements, giving rise to the $\beta$-relaxation. This broader $\beta$ relaxation was observed in the range of $50-170{ }^{\circ} \mathrm{C}$ and has been assigned in the case of polyoxadiazoles, ${ }^{53}$ polyimides, ${ }^{52}$ thermally rearranged polyimides, ${ }^{49}$ and polytriazole asymmetric membranes ${ }^{26}$ to the local mobility of the main polymeric backbone, which can encompass segments that oscillate in a correlated manner. Taking into consideration the most flexible bonds in the PTA-OH, we believe that the $\gamma$-relaxation might include movements of the phenyl side groups bearing $\mathrm{OH}$ groups, while $\beta$-relaxation mobility could be linked to flexibility of quaternary C-phenyl bond in the main chain and involves local rotation, bending, or stretching of the polymer backbone, which also moves in a coupled manner with lateral groups. Therefore, the analysis of these local transitions provides additional insights into the ZIF-polymer interactions. Changes of the two relaxations are observed for the ZIF-8 mixed-matrix membranes (Figures 7 and S12). Broader peaks indicate that the system is less homogeneous with the addition of filler. For the films with 5 and 10 wt \% ZIF-8, the $\gamma$ relaxation was shifted to slightly higher temperatures and was detected with lower intensity, suggesting a reduced mobility of pendant polymeric groups. For higher ZIF-8 contents (15 and 20 wt \%), $\gamma$-relaxation could not be detected in the temperature range covered by the available DMA equipment (down to $-90{ }^{\circ} \mathrm{C}$ ), but the increased tail of $E^{\prime \prime}(T)$ at these temperatures can be a hint that the relaxation is located at lower temperatures.

A similar trend was observed for $\beta$-relaxation, which shifted to higher temperatures for the 5 and 10 wt $\%$ ZIF-8 membranes. Additionally, the relaxation was wider and had a shoulder at a high temperature. This shoulder on the right side of the $\beta$-transition observed in the case of ZIF- 8 composites suggests areas with more limited mobility, which are known to exist at the filler proximity. ${ }^{54,55}$ The wider distribution of relaxation times may be explained by the mobility of the polymer in bulk and at the polymer-ZIF interface and 
potentially can be explained by the polytriazole-ZIF-8 interactions. More than this, this relaxation has a dual character in the case of 15 wt \% ZIF- 8 membrane. The activation energy for the two relaxations estimated from the Arrhenius plot in Figure $7 \mathrm{~b}$ and reported in Table 1 offers

Table 1. Activation Energy of the Relaxations Measured by DMA for PTA-OH and Composite Films with ZIF-8

\begin{tabular}{|lcc|}
\hline \multicolumn{1}{|c}{ sample } & $E_{\mathrm{a}, \gamma}(\mathrm{kJ} / \mathrm{mol})$ & $E_{\mathrm{a}, \beta}(\mathrm{kJ} / \mathrm{mol})$ \\
\hline PTA-OH & 47.48 & 97.43 \\
5 wt \% ZIF-8 & 67.19 & 184.86 \\
10 wt \% ZIF-8 & 62.28 & 160.92 \\
15 wt \% ZIF-8 & & 92.97 \\
20 wt \% ZIF-8 & & 94.27 \\
\hline
\end{tabular}

further support for these affirmations: $E_{\mathrm{a}}$ increases with the ZIF content between 10 and $15 \mathrm{wt} \%$, while for a higher ZIF-8 content, the $\beta$ relaxation has a lower activation energy. A possible hypothesis is that above this concentration, the high ZIF-8 loading induces a looser packing of the PTA-OH polymer creating a higher free volume, which facilitates the molecular mobility of the polymer. At the same time, the increased mobility would also facilitate the release of solvent molecules trapped in the ZIF- 8 particles and could explain the peaks located around $200{ }^{\circ} \mathrm{C}$. Overall, the mobility increase may impact the performance of separation membranes by increasing the permeability but decreasing the selectivity. The relevance of local mobility probed as by DMA on the membrane performance has been pinpointed before both for solvent $^{26}$ and for gas diffusion applications. ${ }^{49,50,56-58}$ Here, we highlight its importance to understand preferential interactions provided by additional functionalization of polymers like PTA$\mathrm{OH}$ and others developed targeting an improvement of adhesion between fillers and polymer matrix. The DMA validates the observations in solution. The rheology investigation in solution is highly sensitive to the polymer-filler interactions and could be an important tool in developing more effective composites and films particularly in applications like separation membranes for which even the presence of minor cavities might compromise the performance.

\section{CONCLUSIONS}

We investigated the rheology of solutions of hydroxylfunctionalized polytriazole and ZIF-8. We confirmed that rheological measurements are highly sensitive to the interactions between the filler and polymer, even at a low ZIF-8 content. Gelation was observed for 10 wt \% PTA-OH solutions containing 3-15 wt \% (relative to the polymer), while solutions with analogous PTA without $\mathrm{OH}$ functionalization did not gel. These results indicate that rheology could be a simple method to screen new fillers/polymer pairs using small amounts of materials, before investing time and material in the membrane optimization. The investigation was supported by microscopic, spectroscopic, mechanical, and dynamic mechanical experiments. SEM images confirmed the formation of cavities in membranes prepared with PTA without $\mathrm{OH}$, while those prepared with gelling PTA-OH/ ZIF-8 systems had a minimum presence of cavities. FTIR identifies the added filler but was not sensitive to clarify or quantify interactions. NMR confirmed that the $\mathrm{OH}$ groups in PTA are involved in the interaction with ZIF-8. Therefore, we believe that the phenol-imidazole interaction is the main reason for the gelation in this work.

Since a large increase in viscosity was observed even with $3 \%$ filler, rheology seems to be very convenient to identify the best polymer/filler pairs, being more sensitive than spectroscopic or mechanical methods. The application can be easily extended to test new fillers and functionalized polymers.

\section{ASSOCIATED CONTENT}

\section{SI Supporting Information}

The Supporting Information is available free of charge at https://pubs.acs.org/doi/10.1021/acsapm.1c00501.

Literature examples of mixed-matrix membranes prepared with ZIF; detailed experimental procedures; Maxwell and Einstein equations; additional SEM images for 5 wt \% ZIF-8 samples; additional rheological, spectroscopic, and X-ray characterization; and mechanical and additional DMA (PDF)

\section{AUTHOR INFORMATION}

\section{Corresponding Author}

Suzana P. Nunes - Biological and Environmental Science and Engineering Division (BESE), Nanostructured Polymeric Membranes Lab, Advanced Membranes and Porous Materials Center, King Abdullah University of Science and Technology, 23955-6900 Thuwal, Saudi Arabia; 다이.org/0000-0002-3669-138X;

Email: Suzana.nunes@kaust.edu.sa

\section{Authors}

Rebecca Esposito - Biological and Environmental Science and Engineering Division (BESE), Nanostructured Polymeric Membranes Lab, Advanced Membranes and Porous Materials Center, King Abdullah University of Science and Technology, 23955-6900 Thuwal, Saudi Arabia

Valentina Musteata - Biological and Environmental Science and Engineering Division (BESE), Nanostructured Polymeric Membranes Lab, Advanced Membranes and Porous Materials Center, King Abdullah University of Science and Technology, 23955-6900 Thuwal, Saudi Arabia; (1) orcid.org/0000-0002-2535-9066

Stefan Chisca - Biological and Environmental Science and Engineering Division (BESE), Nanostructured Polymeric Membranes Lab, Advanced Membranes and Porous Materials Center, King Abdullah University of Science and Technology, 23955-6900 Thuwal, Saudi Arabia

Complete contact information is available at: https://pubs.acs.org/10.1021/acsapm.1c00501

\section{Author Contributions}

All authors have discussed and approved the manuscript. The authors declare no competing financial interest.

\section{Notes}

The authors declare no competing financial interest.

\section{ACKNOWLEDGMENTS}

This work was funded by King Abdullah University of Science and Technology (KAUST). The authors thank KAUST core lab and particularly Edy Abou-Hamad for helping with the NMR measurements. 


\section{REFERENCES}

(1) Kulprathipanja, S.; Neuzil, R.; Li, N. N. Separation of Fluids by Means of Mixed Matrix Membranes. U.S. Patent US4,740,219 A1988.

(2) Kulprathipanja, S.; Neuzil, R.; Li, N. N. Separation of Gases by Means of Mixed Matrix Membranes. U.S. Patent US5,127,925A1989.

(3) Jia, M.; Peinemann, K.-V.; Behling, R.-D. Molecular sieving effect of the zeolite-filled silicone rubber membranes in gas permeation. $J$. Membr. Sci. 1991, 57, 289-292.

(4) Noble, R. D. Perspectives on mixed matrix membranes. J. Membr. Sci. 2011, 378, 393-397.

(5) Chung, T.-S.; Jiang, L. Y.; Li, Y.; Kulprathipanja, S. Mixed matrix membranes (MMMs) comprising organic polymers with dispersed inorganic fillers for gas separation. Progr. Polym. Sci. 2007, 32, 483507.

(6) Dechnik, J.; Gascon, J.; Doonan, C. J.; Janiak, C.; Sumby, C. J. Mixed-matrix membranes. Angew. Chem., Int. Ed. 2017, 56, 92929310.

(7) Galizia, M.; Chi, W. S.; Smith, Z. P.; Merkel, T. C.; Baker, R. W.; Freeman, B. D. 50th anniversary perspective: polymers and mixed matrix membranes for gas and vapor separation: a review and prospective opportunities. Macromolecules 2017, 50, 7809-7843.

(8) Dechnik, J.; Sumby, C. J.; Janiak, C. Enhancing mixed-matrix membrane performance with metal-organic framework additives. Cryst. Growth Des. 2017, 17, 4467-4488.

(9) Jian, M.; Qiu, R.; Xia, Y.; Lu, J.; Chen, Y.; Gu, Q.; Liu, R.; Hu, C.; $\mathrm{Qu}$, J.; Wang, H.; et al. Ultrathin water-stable metal-organic framework membranes for ion separation. Sci. Adv. 2020, 6, No. eaay3998

(10) Qiu, S.; Xue, M.; Zhu, G. Metal-organic framework membranes: from synthesis to separation application. Chem. Soc. Rev. 2014, 43, 6116-6140.

(11) Knebel, A.; Bavykina, A.; Datta, S. J.; Sundermann, L.; GarzonTovar, L.; Lebedev, Y.; Durini, S.; Ahmad, R.; Kozlov, S. M.; Shterk, G.; et al. Solution processable metal-organic frameworks for mixed matrix membranes using porous liquids. Nat. Mater. 2020, 19, 13461353.

(12) Long, J. R.; Yaghi, O. M. The pervasive chemistry of metalorganic frameworks. Chem. Soc. Rev. 2009, 38, 1213-1214.

(13) Li, H.; Eddaoudi, M.; O’Keeffe, M.; Yaghi, O. M. Design and synthesis of an exceptionally stable and highly porous metal-organic framework. Nature 1999, 402, 276-279.

(14) Zimmerman, C. M.; Singh, A.; Koros, W. J. Tailoring mixed matrix composite membranes for gas separations. J. Membr. Sci. 1997, 137, 145-154.

(15) Lin, R.; Hernandez, B. V.; Ge, L.; Zhu, Z. Metal organic framework based mixed matrix membranes: an overview on filler/ polymer interfaces. J. Mater. Chem. A 2018, 6, 293-312.

(16) Dai, Y.; Johnson, J.; Karvan, O.; Sholl, D. S.; Koros, W. Ultem/ ZIF-8 mixed matrix hollow fiber membranes for $\mathrm{CO} 2 / \mathrm{N} 2$ separations. J. Membr. Sci. 2012, 401, 76-82.

(17) Eiras, D.; Labreche, Y.; Pessan, L. A. Ultem/ZIF-8 mixed matrix membranes for gas separation: transport and physical properties. Mater. Res. 2016, 19, 220-228.

(18) Gao, Y.; Qiao, Z.; Zhao, S.; Wang, Z.; Wang, J. In situ synthesis of polymer grafted ZIFs and application in mixed matrix membrane for CO 2 separation. J. Mater. Chem. A 2018, 6, 3151-3161.

(19) Benzaqui, M.; Semino, R.; Menguy, N.; Carn, F.; Kundu, T.; Guigner, J.-M.; McKeown, N. B.; Msayib, K. J.; Carta, M.; MalpassEvans, R.; et al. Toward an understanding of the microstructure and interfacial properties of PIMs/ZIF-8 mixed matrix membranes. ACS Appl. Mater. Interfaces 2016, 8, 27311-27321.

(20) Li, G.; Si, Z.; Cai, D.; Wang, Z.; Qin, P.; Tan, T. The in-situ synthesis of a high-flux ZIF-8/polydimethylsiloxane mixed matrix membrane for n-butanol pervaporation. Sep. Purif. Technol. 2020, 236, No. 116263

(21) Ordonez, M. J. C.; Balkus, K. J., Jr.; Ferraris, J. P.; Musselman, I. H. Molecular sieving realized with ZIF-8/Matrimid mixed-matrix membranes. J. Membr. Sci. 2010, 361, 28-37.
(22) Shi, G. M.; Yang, T.; Chung, T. S. Polybenzimidazole (PBI)/ zeolitic imidazolate frameworks (ZIF-8) mixed matrix membranes for pervaporation dehydration of alcohols. J. Membr. Sci. 2012, 415, 577586.

(23) Zhang, C.; Dai, Y.; Johnson, J. R.; Karvan, O.; Koros, W. J. High performance ZIF-8/6FDA-DAM mixed matrix membrane for propylene/propane separations. J. Membr. Sci. 2012, 389, 34-42.

(24) Fan, Y.; Yu, H.; Xu, S.; Shen, Q.; Ye, H.; Li, N. Zn (II)modified imidazole containing polyimide/ZIF-8 mixed matrix membranes for gas separations. J. Membr. Sci. 2020, 597, No. 117775.

(25) Chisca, S.; Duong, P.; Emwas, A.-H.; Sougrat, R.; Nunes, S. P. Crosslinked copolyazoles with a zwitterionic structure for organic solvent resistant membranes. Polym. Chem. 2015, 6, 543-554.

(26) Chisca, S.; Marchesi, T.; Falca, G.; Musteata, V.-E.; Huang, T.; Abou-Hamad, E.; Nunes, S. P. Organic solvent and thermal resistant polytriazole membranes with enhanced mechanical properties cast from solutions in non-toxic solvents. J. Membr. Sci. 2020, 597, No. 117634

(27) Chisca, S.; Falca, G.; Musteata, V. E.; Boi, C.; Nunes, S. P. Crosslinked polytriazole membranes for organophilic filtration. J. Membr. Sci. 2017, 528, 264-272.

(28) Flory, P. J. Principles of Polymer Chemistry; Cornell University Press, 1953

(29) Stockmayer, W. H. Theory of molecular size distribution and gel formation in branched-chain polymers. J. Chem. Phys. 1943, 11, $45-55$.

(30) De Gennes, P. Conformation of a polymer chain in certain mixed solvents. J. Phys. Lett. 1976, 37, 59-61.

(31) Nunes, S.; Wolf, B. On the cooccurrence of demixing and thermoreversible gelation of polymer solutions. 3. Overall view. Macromolecules 1987, 20, 1952-1957.

(32) Djabourov, M. Gelation-A review. Polym. Int. 1991, 25, 135143.

(33) Maab, H.; Al Saadi, A.; Francis, L.; Livazovic, S.; Ghafour, N.; Amy, G. L.; Nunes, S. P. Polyazole hollow fiber membranes for direct contact membrane distillation. Ind. Eng. Chem. Res. 2013, 52, 1042510429 .

(34) Graessley, W. W. The Entanglement Concept in Polymer Rheology. In The Entanglement Concept in Polymer Rheology; Springer, 1974; pp 1-179.

(35) Jelich, L.; Nunes, S.; Paul, E.; Wolf, B. On the cooccurrence of demixing and thermoreversible gelation of polymer solutions. 1 . Experimental observations. Macromolecules 1987, 20, 1943-1947.

(36) Chassenieux, C.; Nicolai, T.; Benyahia, L. Rheology of associative polymer solutions. Curr. Opin. Colloid Interface Sci. 2011, 16, 18-26.

(37) Pryamitsyn, V.; Ganesan, V. Mechanisms of steady-shear rheology in polymer-nanoparticle composites. J. Rheol. 2006, 50, 655-683.

(38) Basma, N.; Cullen, P.; Clancy, A.; Shaffer, M.; Skipper, N.; Headen, T.; Howard, C. The liquid structure of the solvents dimethylformamide (DMF) and dimethylacetamide (DMA). Mol. Phys. 2019, 117, 3353-3363.

(39) Calle, M.; Doherty, C. M.; Hill, A. J.; Lee, Y. M. Cross-linked thermally rearranged poly (benzoxazole-co-imide) membranes for gas separation. Macromolecules 2013, 46, 8179-8189.

(40) Gao, S.; Hou, J.; Deng, Z.; Wang, T.; Beyer, S.; Buzanich, A. G.; Richardson, J. J.; Rawal, A.; Seidel, R.; Zulkifli, M. Y.; et al. Improving the acidic stability of zeolitic imidazolate frameworks by biofunctional molecules. Chem 2019, 5, 1597-1608.

(41) Moulin, A.; Bibian, M.; Blayo, A.-L.; El Habnouni, S.; Martinez, J.; Fehrentz, J.-A. Synthesis of 3, 4, 5-Trisubstituted-1, 2, 4-triazoles. Chem. Rev. 2010, 110, 1809-1827.

(42) El Ghomari, M.; Mokhlisse, R.; Laurence, C.; Le Questel, J. Y.; Berthelot, M. Basicity of azoles: complexes of diiodine with imidazoles, pyrazoles and triazoles. J. Phys. Org. Chem. 1997, 10, 669-674.

(43) Epley, T. D.; Drago, R. S. Calorimetric studies on some hydrogen-bonded adducts. J. Am. Chem. Soc. 1967, 89, 5770-5773. 
(44) Fairen-Jimenez, D.; Moggach, S.; Wharmby, M.; Wright, P.; Parsons, S.; Duren, T. Opening the gate: framework flexibility in ZIF8 explored by experiments and simulations. J. Am. Chem. Soc. 2011, $133,8900-8902$.

(45) Ma, K.; Idrees, K. B.; Son, F. A.; Maldonado, R.; Wasson, M. C.; Zhang, X.; Wang, X.; Shehayeb, E.; Merhi, A.; Kaafarani, B. R.; et al. Fiber composites of metal-organic frameworks. Chem. Mater. 2020, 32, 7120-7140.

(46) Polak-Kraśna, K.; Dawson, R.; Holyfield, L. T.; Bowen, C. R.; Burrows, A. D.; Mays, T. J. Mechanical characterisation of polymer of intrinsic microporosity PIM-1 for hydrogen storage applications. J. Mater. Sci. 2017, 52, 3862-3875.

(47) Ma, X.; Swaidan, R. J.; Wang, Y.; Hsiung, C.-e.; Han, Y.; Pinnau, I. Highly compatible hydroxyl-functionalized microporous polyimide-ZIF-8 mixed matrix membranes for energy efficient propylene/propane separation. ACS Appl. Nano Mater. 2018, 1, $3541-3547$.

(48) Comer, A. C.; Kalika, D. S.; Rowe, B. W.; Freeman, B. D.; Paul, D. R. Dynamic relaxation characteristics of Matrimid polyimide. Polymer 2009, 50, 891-897.

(49) Comer, A. C.; Ribeiro, C. P.; Freeman, B. D.; Kalakkunnath, S.; Kalika, D. S. Dynamic relaxation characteristics of thermally rearranged aromatic polyimides. Polymer 2013, 54, 891-900.

(50) Mahdi, E. M.; Tan, J.-C. Mixed-matrix membranes of zeolitic imidazolate framework (ZIF-8)/Matrimid nanocomposite: Thermomechanical stability and viscoelasticity underpinning membrane separation performance. J. Membr. Sci. 2016, 498, 276-290.

(51) Menard, K. P. Dynamic Mechanical Analysis A Practical Introduction, 2nd ed.; CRC Press: Boca Raton, 2008.

(52) Chisca, S.; Musteata, V. E.; Sava, I.; Bruma, M. Dielectric behavior of some aromatic polyimide films. Eur. Polym. J. 2011, 47, 1186-1197.

(53) Damaceanu, M.-D.; Musteata, V.-E.; Cristea, M.; Bruma, M. Viscoelastic and dielectric behaviour of thin films made from siloxanecontaining poly(oxadiazole-imide)s. Eur. Polym. J. 2010, 46, 10491062.

(54) Bashir, M. A. Use of Dynamic Mechanical Analysis (DMA) for Characterizing Interfacial Interactions in Filled Polymers. Solids 2021, 2, $108-120$.

(55) Bailey, E. J.; Winey, K. I. Dynamics of polymer segments, polymer chains, and nanoparticles in polymer nanocomposite melts: A review. Progr. Polym. Sci. 2020, 105, No. 101242.

(56) Huang, S.-H.; Hu, C.-C.; Lee, K.-R.; Liaw, D.-J.; Lai, J.-Y. Gas separation properties of aromatic poly(amide-imide) membranes. Eur. Polym. J. 2006, 42, 140-148.

(57) Iyer, K. A. Chain mobility, secondary relaxation, and oxygen transport in terephthalate copolyesters with rigid and flexible cyclic diols. Polymer 2017, 129, 117-126.

(58) Butnaru, I.; Constantin, C.-P.; Asandulesa, M.; WolińskaGrabczyk, A.; Jankowski, A.; Szeluga, U.; Damaceanu, M.-D. Insights into molecular engineering of membranes based on fluorinated polyimide-polyamide miscible blends which do not obey the trade-off rule. Sep. Purif. Technol. 2020, 233, No. 116031. 